\title{
Fluid shear stress induces cancer stem cell-like phenotype in MCF7 breast cancer cell line without inducing epithelial to mesenchymal transition
}

\author{
URSULA L. TRIANTAFILLU ${ }^{1}$, SEUNGJO PARK ${ }^{1}$, NIKKI L. KLAASSEN ${ }^{2}$, \\ ANDREW D. RADDATZ ${ }^{1}$ and YONGHYUN KIM ${ }^{1}$ \\ ${ }^{1}$ Department of Chemical and Biological Engineering, The University of Alabama, Tuscaloosa, AL; \\ ${ }^{2}$ Department of Chemical Engineering, Kansas State University, Manhattan, KS, USA
}

Received September 5, 2016; Accepted January 20, 2017

DOI: 10.3892/ijo.2017.3865

\begin{abstract}
Metastasis is the leading cause of cancer deaths due to the spread of cancer cells through the blood vessels and the subsequent formation of secondary tumors. Metastasizing cancer cells in the human vasculature are called circulating tumor cells (CTCs) and are characterized to express the epithelial cell adhesion molecule (EpCAM). They are further known to survive physiological fluid shear stress (FSS) conditions. However, the effect of FSS on CTC molecular phenotype, such as the epithelial to mesenchymal transition (EMT) and cancer stem cell (CSC) expression, has not been extensively studied. Here, CTCs in FSS are evaluated in an in vitro model system. MCF7 and MDA-MB-231 breast cancer cell lines were grown in adherent and suspension culture media. The cell lines were tested for EMT and CSC genetic and protein markers using qRT-PCR and flow cytometry, respectively. Suspension cells showed a significantly increased EMT signature compared to adherent cells $(p<0.05)$, suggesting that they model cells detaching from primary tumors in vivo. Upon application of FSS, MCF7 and MDA-MB-231 cells did not show a significant change in EMT expression ( $p>0.05)$, but there was a statistically significant increase of the CSC population in MCF7 suspension cultures $(\mathrm{p}<0.05)$. These results with MCF7 suggest that CTCs can be modeled in vitro as non-adherent cancer cells in FSS and that they show an increased CSC-like signature
\end{abstract}

Correspondence to: Professor Yonghyun Kim, Department of Chemical and Biological Engineering, The University of Alabama, Box 870203, Tuscaloosa, AL 35487-0203, USA

E-mail: ykim@eng.ua.edu

Abbreviations: FSS, fluid shear stress; EMT, epithelial to mesenchymal transition; CSC, cancer stem cell; CTC, circulating tumor cell

Key words: fluid shear stress, metastasis, circulating tumor cell, epithelial to mesenchymal transition, epithelial cell adhesion molecule, cancer stem cell during circulation, providing new insights to the importance of CSC-targeting strategies when treating metastatic patients.

\section{Introduction}

Most cancer deaths are not due to primary tumors, but instead due to metastasis (1). Metastasis arises from biological and physical changes of cancer cells in the primary tumor based on the following process: i) detachment from primary tumor, ii) penetration into vasculature (intravasation), iii) circulation in vasculature, iv) penetration out of vasculature to surrounding organ tissue (extravasation), and v) seeding and secondary tumor formation. In order for cells to undergo the first step of detachment from the primary tumor, cells undergo a process known as epithelial to mesenchymal transition (EMT). This motility allows for the cells to move into the blood vessels in the process known as intravasation. After intravasation, the cancer cells are able to travel throughout the body either to localized or distant sites throughout the circulation. They are therefore dubbed 'circulating tumor cells' (CTCs) before undergoing either adhesion to the blood vessel walls or entrapment into smaller diameter blood vessels.

Detection of cancer metastasis is challenging since most metastasizing cancers are only detected after a sizable formation of secondary tumors. Early detection technology for metastasis has focused on identifying CTCs obtained from blood samples. These blood samples are tested for specific cell surface markers to identify the CTCs. Most clinically used CTC detection devices, such as CellSearch ${ }^{\circledR}$, rely on antibody-based detection of cytokeratins as well as EpCAM, a widely accepted CTC marker due to its expression in epithelial cancers (2-5). Despite the advances in CTC detection, detecting metastasis is still challenging due to the low detection of CTCs, false positives, and changing nature of the cells.

Although metastasis involves progression via blood circulation, the effects of blood circulation on breast cancer CTCs have not been extensively studied. CTCs inherently experience fluid shear stress (FSS) in the blood. In the tumor, cells experience interstitial flow with an average shear stress of $0.1 \mathrm{dyn} / \mathrm{cm}^{2}$, but CTCs in blood flow experience a higher 
FSS range that averages from $0.5-30.0 \mathrm{dyn} / \mathrm{cm}^{2}$ that are calculated by using the Hagen-Poiseuille equation (6). Survival of CTCs in blood circulation makes them unique from other non-hematological cells. It has been observed that cancer cells, including breast cancer cells, have a higher resistance to FSS than non-cancerous cells (7). The resistance of CTCs in FSS is believed to be due to these cells being of cancer stem cell (CSC) origin $(8,9)$.

CSCs are a subpopulation of cancer cells that have stem cell-like properties, which allows them to self-renew and differentiate into bulk tumor cells. The CSC population is therefore believed to be responsible for tumor initiation and relapse $(10,11)$. CSCs have been identified in many types of cancers, including breast cancer, in which they are identified as $\mathrm{CD}_{4} 4^{+} / \mathrm{CD} 24^{-/ \text {low }} / \mathrm{ALDH}^{+}$cells $(12,13)$. CSC population in in vitro conditions are affected by the culture conditions (14). Typically, breast cancer cell lines are cultured in tissue culture flasks adherently, but recent studies show that mammosphere cultures better retain the CSC population of breast cancer (15). However, the characteristics of CSCs in FSS conditions are understudied.

Therefore, in this study we mimicked the biological FSS on normal breast and breast cancer cells with an in vitro fluid flow model. This model allowed us to assess how FSS affects the important phenotypes such as EMT, CTC, and CSC marker expression that arise during metastasis. Our model provides a new understanding of the relationship between CTC- and CSC-signatures for breast cancer cells in FSS.

\section{Materials and methods}

Cell culture. Three breast cancer cell lines MCF7, MDA-MB231, and SKBR3 (ATCC, Manassas, VA, USA) were cultured in phenol-red free Dulbecco's modified Eagle's medium (DMEM) (Gibco, Grand Island, NY, USA) supplemented with 1\% L-glutamine (Life Technologies, Carlsbad, CA, USA), 10\% Fetal Bovine Serum (FBS) (Gibco), and 1\% penicillin/streptomycin (Corning, Manassas, VA, USA). The non-cancerous breast epithelial cell line 184A1 (ATCC) was cultured in mammary epithelial basal medium (MEBM) (Lonza) supplemented with $0.005 \mathrm{mg} / \mathrm{ml}$ transferrin, $1 \mathrm{ng} / \mathrm{ml}$ cholera toxin, and $1 \%$ penicillin/streptomycin (Corning). All cell lines were cultured with their respective recommended protocols. MCF7 and MDA-MB-231 mammospheres were formulated by culture in phenol-red free DMEM: Nutrient Mixture F12 (DMEM/F12) (Gibco) supplemented with 1X B27 (Gibco), $5 \mathrm{mg} / 1$ insulin (MBL International Corp., Woburn, MA, USA), $20 \mu \mathrm{g} / 1$ bFGF (Shenandoah Inc., Warwick, PA, USA), $20 \mu \mathrm{g} / 1 \mathrm{EFG}$ (Shenandoah Inc.), $1 \%$ penicillin/streptomycin (Corning), $0.5 \mathrm{mg} / \mathrm{l}$ hydrocortisone (Sigma Aldrich, St. Louis, MO, USA), and $2.5 \mathrm{mM}$ L-glutamine (Life Technologies) modified from (16).

Fluid shear stress (FSS) and flow apparatus. A Fusion 200 Series syringe pump (Chemyx, Stafford, TX, USA) was used in applying FSS on the cell lines in Polyether ether-ketone (PEEK) tubing (IDEX Health \& Science) with a $46 \mathrm{~cm}$ length and $125 \mu \mathrm{m}$ inner diameter. A $3 \mathrm{ml}$ syringe (BD Sciences) was attached to the PEEK tubing by a leur-lok and leur tight fitting (IDEX Health \& Science) (Fig. 1A).
FSS was calculated using Hagen-Poiseuille's equation for blood flow. A FSS of $60 \mathrm{dyn} / \mathrm{cm}^{2}$ was used in this experiment which correlates to the higher FSS cells experience during blood flow. The time of FSS ranged to approximately $30 \mathrm{~min}$ for a $2 \mathrm{ml}$ volume. The fluid viscosity of the cell media, $1.0 \times 10^{-3} \mathrm{~kg} / \mathrm{m} / \mathrm{sec}$, was used for $\mu$. The inner diameter of the tubing was used for $\mathrm{d}$. The volumetric flow rate was calculated to be $68.9 \mu \mathrm{l} / \mathrm{min}$ for a FSS of $60 \mathrm{dyn} / \mathrm{cm}^{2}$.

All experiments were performed at ambient conditions in a biological hood for sterile conditions. Cells were collected in PBS after passaging at $5 \times 10^{5}$ cells $/ \mathrm{ml}$ and drawn up manually into the syringe. The leur-lok, tight fitting, and PEEK tubing were attached to the syringe. The syringe was placed in the syringe pump and set at the calculated flow rate. Dispelled cells were collected in open $1.5 \mathrm{ml}$ centrifuge tubes. The cells were centrifuged down and were either evaluated immediately for flow cytometry (within 5 min of collection) or flash frozen as qRT-PCR samples for later RNA isolation.

Fluid flow modeling. COMSOL Multiphysics 4.3 with the computational fluid dynamics (CFD) module (Burlington, MA, USA) was used to model the experimental set-up with flow in the syringe and tubing. Water fluidic settings was used and the following parameters were specified: $8.66 \mathrm{~mm}$ for the syringe diameter, $6.5 \mathrm{~cm}$ for the syringe length, $1 \mathrm{~cm}$ for nozzle of syringe, $125 \mu \mathrm{m}$ for the diameter of the tubing, $45 \mathrm{~cm}$ for the length of the tubing, and non-slip boundary conditions. These parameters match the experimental setup described above. The velocity profile and FSS profiles were obtained for the given set-up.

Cell viability experiment. Cell viability of the four cell lines were tested immediately (within $5 \mathrm{~min}$ ) after applying $20 \mathrm{dyn} / \mathrm{cm}^{2}$ and $60 \mathrm{dyn} / \mathrm{cm}^{2} \mathrm{FSS}$. Viability of non-sheared cells (i.e., $0 \mathrm{dyn} / \mathrm{cm}^{2}$ ) was measured as control. Cells were inserted into a $3 \mathrm{ml}$ syringe at a density of $3 \times 10^{5}$ cells $/ \mathrm{ml}$ and placed in the fluid flow set-up as previously mentioned. Cells were collected at $10 \mathrm{~min}$ time intervals for three time periods. Cell viability was tested using trypan blue (Amresco, Solon, $\mathrm{OH}$, USA) to stain for a live/dead cell count in the T20 automated cell counter (Bio-Rad).

$q R T-P C R$. Primers were designed by retrieving nucleotide sequences from NCBI gene database as follows: $C D H 1$ forward: CCT CCT TGG CTC AAA CGA CA, reverse: TCC ACC ACA GTG TTC AGT CG; $C D H 2$ forward: TGC CTC TGG TGA TGA TAC GC, reverse: GTA CCA AGA AGT GCC AGC CT; EpCAM forward: GTG GTT GTG GTG ATA GCA GTT G, reverse: ACT TGT TCT GTA CTC ACC AGC; VIM forward: AGG TTC AGG TTT CAT TCA TGC C, reverse: AGT TGG CTG TGT GTA CTG CT; $S L U G$ forward: TCT GGT TGT GGT ATG ACA GGC, reverse: TGT GTG TAT ACT TGC GTG TGG A; SNAIL forward: CTG GGC TTG CTG TTC TCA TTC, reverse: CTG CTT TGC CCA TCT GCT TAG; NANOG forward: AAT ACC TCA GCC TCC AGC AGA TG, reverse: TGC GTC ACA CCA TTG CTA TTC TTC; and OCT4 forward: GAG AAC CGA GTG AGA GGC AAC C, reverse: CAT AGT CGC TGC TTG ATC GCT TG. ACTB was used as a housekeeping gene with the primer sequences for ACTB forward: TCG TCG CCC ACA TAG 
GAA and reverse: AGG GCT TCT TGT CCT TTC CTTC. $G A P D H$ was used as a housekeeping gene with the primer sequences for GAPDH forward: AGA GCA CAA GAG GAA GAG AGA GAC and reverse: AGC ACA GGG TAC TTT ATT GAT GGT. Primers were synthesized by Eurofins Genomics (Huntsville, AL, USA).

RNA isolation was performed using GeneJet RNA Purification kit (Thermo Fisher Scientific, Pittsburg, PA, USA) using the manufacturer's protocol for mammalian cultured cells. RNA quantification was performed using Qubit RNA HS assay kit and Qubit 2.0 Fluorometer (Life TechnologiesInvitrogen, Carlsbad, CA, USA) and by using NanoDrop 2000 according to manufacturer's protocol (Thermo Scientific, Wilmington, DE, USA). Complementary DNA (cDNA) was synthesized using qScript cDNA SuperMix (Quanta Biosciences, Gaithersburg, MD, USA) and Mastercycler Nexus Gradient (Eppendorf, Hauppauge, NY, USA) according to the manufacturer's protocol. Real-time PCR was performed using PerfeCTa SYBR Green Fast Mix (Quanta Biosciences) according to the manufacturer's protocol. Eco Real-time PCR System (Illumina) and StepOnePlus Real-time PCR System (Thermo Fisher Scientific) were used as the qRT-PCR instrument. EcoStudy software (v4.0) and StepOne Software (v2.3) were used for the data analysis using the $\Delta \Delta \mathrm{Ct}$ method (17).

Flow cytometry. Flow cytometry was performed using BD Accuri C6 Flow Cytometer (BD Biosciences, San Jose, CA, USA). EpCAM mouse anti-human IgG1 FITC conjugated antibody was used for epithelial marker expression (BD Bioscience, cat. \# 347197). Antibodies for breast CSC marker expression included primary antibody CD24 mouse IgG1 biotin anti-human, secondary antibody anti-biotin PE mouse IgG1 (Miltenyi Biotec, cat. \# 130-098-902 and 130-090756, respectively), and CD44 mouse anti-human IgG2b APC conjugated antibody (BD Biosciences, cat. \# 559942) according to manufacturer's protocol.

Limiting dilution assay. Limiting dilution assay (LDA) was performed on MCF7 and MDA-MB-231 suspension cells under static and FSS conditions. LDAs were seeded in 96-well plates at concentrations of $50,20,10,5$, and 2 cells per well ( $\mathrm{n}=20$ for each seeding concentration). Cells were cultured for 10 days with feeding every 4 days. Wells were analyzed at day 10 for sphere formation. Sphere formation was determined as spheres with diameters $\geq 50 \mu \mathrm{m}$. Significant statistical difference of samples were determined by a $95 \%$ confidence interval (CI). Clonogenicity index was defined as slope \pm SE of the LDA linear regression (18).

Western blotting. Adherent, suspension static, and suspension FSS MCF7 and MDA-MB-231 cells were lysed in NP40 buffer (Amresco) with 0.5 M EDTA (G-Bioscience, St. Louis, MO, USA), protease inhibitor (G-Bioscience), phosphatase inhibitor (Sigma), and nuclease (Thermo Scientific). Protein concentrations were measured by BSA (G-Bioscience). Protein was loaded for western blotting. Skim milk (5\%) (Criterion) was used for membrane blocking and then stained with following antibodies: ACTB (GeneTex, cat. \# GTX26276), NANOG (Thermo Scientific, cat. \# MA1-017), and OCT4 (Thermo Scientific, cat. \# MA5-15736). Western blots were developed in a digital imager (Bio-Rad ChemiDoc MP) and normalized to ACTB. Protein expression was quantified using Image Lab software (Bio-Rad).

Statistical analysis. Results were analyzed using Student's two tailed t-test and ANOVA with equal or unequal variance in Minitab 17 (Minitab Inc., State College, PA, USA). F-test and Levene's test were used to determine equal or unequal variance prior to t-test and ANOVA. Differences with p-values $<0.05$ were considered significant.

\section{Results}

Breast epithelial and cancer cells have varying tolerance to FSS. Computational modeling of the fluid flow was generated based on the experimental set-up of an in vitro model that mimics flow in the blood vessels (Fig. 1A). COMSOL modeling of the experimental set-up showed that fluid flow becomes fully developed laminar flow at $0.05 \mathrm{~mm}$ past the entrance into the tubing, which correlates to $0.01 \%$ of the length of the tubing used in our set-up (Fig. 1B). Therefore, entry effects in the tubing were considered insignificant. A fluid flow rate of $23.0 \mu \mathrm{l} / \mathrm{min}$ and $68.9 \mu \mathrm{l} / \mathrm{min}$ was calculated using the HagenPoiseuille equation for 20 and $60 \mathrm{dyn} / \mathrm{cm}^{2}$ of FSS, respectively. COMSOL modeling further demonstrated that the FSS in the syringe is negligible compared to the FSS in the tubing (Fig. 1C). COMSOL modeling therefore confirmed that our set-up could be used to apply FSS in a controlled manner.

FSS has the potential to detrimentally affect cells and even cause cell death. Therefore, cell viability was evaluated in our experimental set-up under conditions of FSS. Physiological FSS values of 20 and $60 \mathrm{dyn} / \mathrm{cm}^{2}$ were tested on the 184A1, MCF7, SKBR3, and MDA-MB-231 cell lines. Cell viability under FSS was normalized to the control conditions of the cell lines at $0 \mathrm{dyn} / \mathrm{cm}^{2}$. The breast epithelial cell line 184A1 experienced a significant decrease in cell viability with only half of the 184A1 cells surviving under FSS conditions. The MCF7 and MDA-MB-231 cell line viability continued to remain high under FSS conditions (Fig. 1D).

Breast cancer cell lines have different baseline EMT gene expressions. EpCAM (CTC marker), CDH1 (E-cadherin; epithelial marker), $\mathrm{CDH} 2$ (N-cadherin; mesenchymal marker), VIM, SLUG, and SNAIL (mesenchymal markers), expression in 184A1, MCF7, SKBR3, and MDA-MB-231 were examined (Fig. 2) (19-21). One of the most noted features of metastasis has been the transition of cells from an epithelial phenotype to a mesenchymal phenotype through EMT. The CDH1 epithelial marker, which is involved in epithelial cell adhesion, had higher expression in the MCF7 and 184A1 cell lines (Fig. 2). The more invasive MDA-MB-231 cell line had significantly higher gene expression of $\mathrm{CDH} 2$ and VIM ( $<<0.05$ and $p<0.01$, respectively). This correlates to $\mathrm{CDH} 2$ and VIM being mesenchymal markers involved in invasion and migration (22). EpCAM was expressed significantly higher in the MCF7 and SKBR3 breast cancer cell lines ( $\mathrm{p}<0.05$ and $\mathrm{p}<0.01$, respectively). Moreover, 184A1 breast epithelial cell line had low EpCAM expression. EpCAM expression is not solely used as an EMT marker, but instead is commonly used as a CTC marker (23). As a 
A

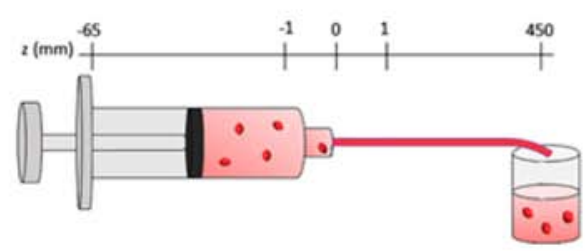

B

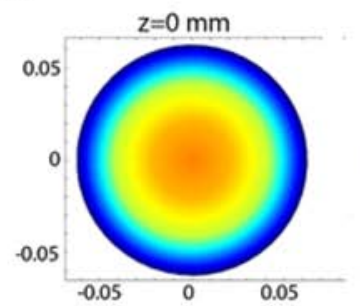

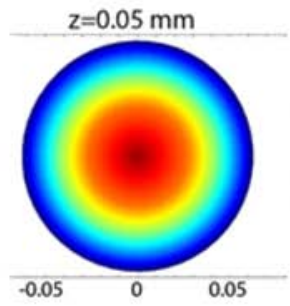

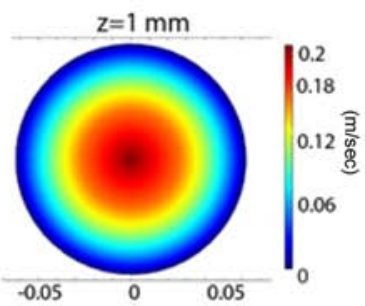

\section{C}
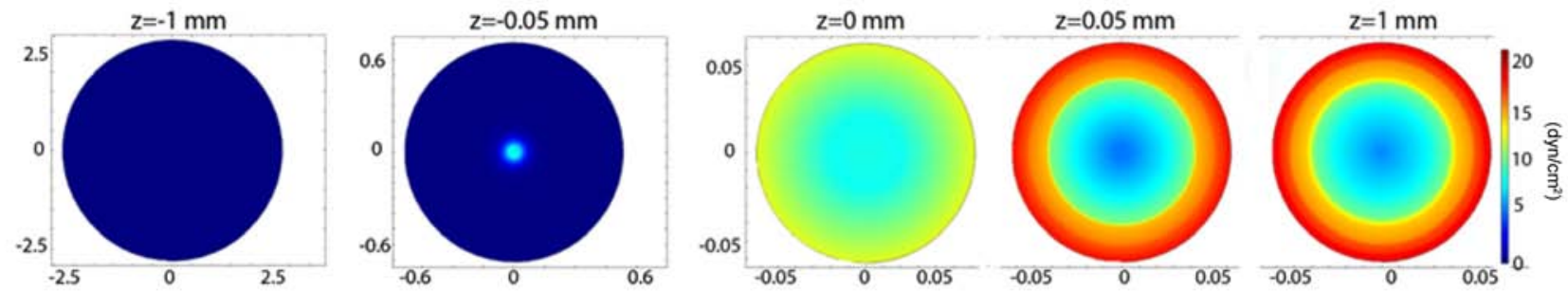

D

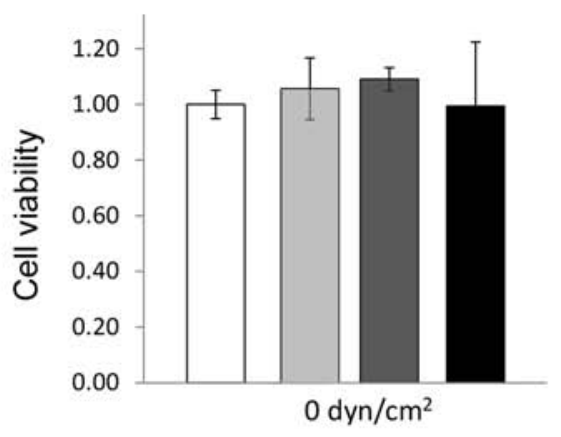

$\square 184 \mathrm{~A} 1$

MCF7

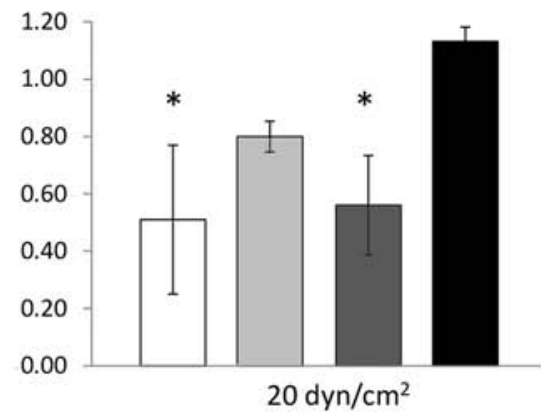

SKBR3

MDA-MB-231

Figure 1. The experimental set-up, COMSOL modeling, and cell viability during fluid flow. (A) Schematic of experimental set-up of fluid flow experiment using syringe and tubing. Axis represents distance $(\mathrm{mm})$ from the syringe-tubing interface $(\mathrm{z}=0)$. (B) COMSOL modeling of velocity profiles in the tubing at $60 \mathrm{dyn} / \mathrm{cm}^{2}$ fluid shear stress (FSS). Left $(\mathrm{z}=0 \mathrm{~mm})$ : Velocity profile at entry of the tubing with a maximum velocity of $0.15 \mathrm{~m} / \mathrm{sec} ; \mathrm{middle}(\mathrm{z}=0.05 \mathrm{~mm})$ : Velocity profile at $0.05 \mathrm{~mm}$ into the tubing with a maximum velocity of $0.19 \mathrm{~m} / \mathrm{sec}$; and right $(\mathrm{z}=1 \mathrm{~mm})$ : Velocity profile at $1 \mathrm{~mm}$ into the tubing with a maximum velocity of $0.20 \mathrm{~m} / \mathrm{sec}$. (C) COMSOL modeling of FSS profiles in the syringe (left; $\mathrm{z}=-1 \mathrm{~mm}$ ), at the syringe-tubing interface (middle; $\mathrm{z}=-0.05 \mathrm{~mm}$ ), and inside the tubing (right; $z=0,0.05$, and $1 \mathrm{~mm}$ ). (D) The viability of breast epithelial cell line 184A1 and breast cancer cell lines SKBR3, MCF7, and MDAMB-231 under $0 \mathrm{dyn} / \mathrm{cm}^{2}$ was compared to $20 \mathrm{dyn} / \mathrm{cm}^{2}$ of FSS (mean $\pm \mathrm{SD} ; \mathrm{n}=3 ;{ }^{*} \mathrm{p}<0.05$ indicates the statistical significant difference between cell lines at 20 dyne $/ \mathrm{cm}^{2}$ from 0 dyne $/ \mathrm{cm}^{2}$ ).

CTC marker, EpCAM is seen to be highly expressed in cancer cells compared to non-cancerous epithelial cells allowing for epithelial based cancer detection. Therefore, variable gene expression of the cell lines was due to their different cellular characteristics. The MCF7 and MDA-MB-231 cell lines were used for further studies due to their difference in gene expression and higher cell viability under FSS.

Suspension culture induces EMT gene expression in MCF7. Since cells typically undergo EMT as the first steps towards metastasis, EMT gene expression was examined with the expectation of an increase in mesenchymal gene expression and a decrease in epithelial gene expression. MCF7 experienced no significant expression change under FSS conditions in comparison to suspension static culture (Fig. 3; p>0.05). Furthermore, MDA-MB-231 had no significant changes in gene expression between the adherent and suspension cultures
( $p>0.05$ ). On the other hand, MCF7 suspension culture cells showed a significant increase in gene expression of EpCAM, $\mathrm{CDH} 1$, and $\mathrm{CDH} 2$ compared to the adherent culture cells $(\mathrm{p}<0.001, \mathrm{p}<0.1$, and $\mathrm{p}<0.5$, respectively). The largest increase in gene expression from the adherent to suspension cultures was seen with CDH2 (20- to 30-fold increase; Fig. 3). EpCAM and $\mathrm{CDH} 1$ expression change was more modest (approximately 8- and 4-fold increase, respectively). These results suggest that there is a strong EMT induction when culturing MCF7 in suspension culture media and that FSS does not negatively impact this induction in MCF7.

MCF7 is more susceptible to changes in CSC expression. To assess clonogenicity, limiting dilution assays (LDAs) were performed on MCF7 and MDA-MB-231 suspension static and suspension FSS cells (Fig. 4). We observed a statistically significant increase in clonogenicity upon applying FSS to 

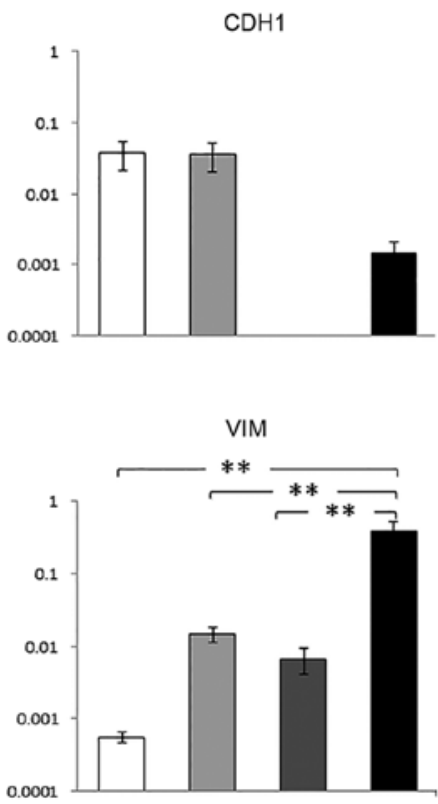

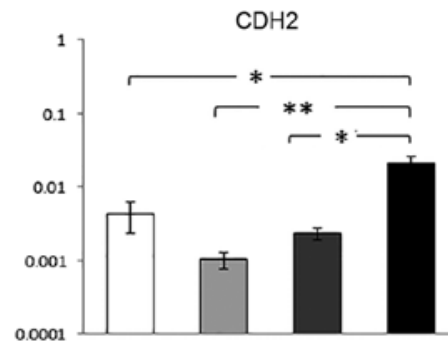

SLUG

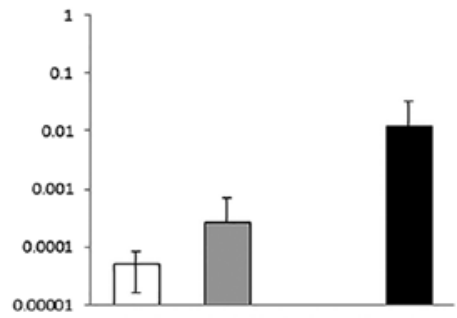

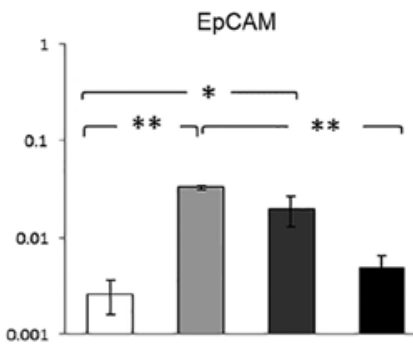

SNAIL

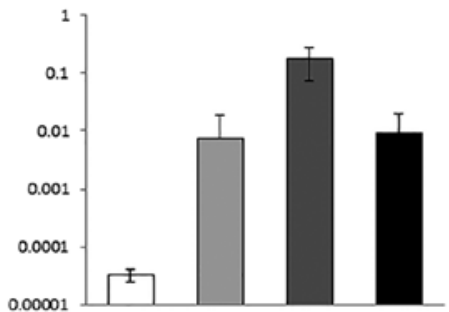

$\square 184 \mathrm{~A} 1 \square \mathrm{MCF7} \square \mathrm{SKBR3} \square \mathrm{MDA}-\mathrm{MB}-231$

Figure 2. Gene expression of breast cancer cells is dependent on the cell line. A baseline comparison of breast epithelial and breast cancer cell lines was performed using qRT-PCR. The breast epithelial cell line 184A1 and breast cancer cell lines MCF7, SKBR3, and MDA-MB-231 were tested for CDH1 (E-cadherin), CDH2 (N-cadherin), EpCAM, VIM, SLUG, and SNAIL gene expression. EpCAM gene expression was significantly upregulated in MCF7 and the mesenchymal markers CDH2 and VIM were significantly upregulated in MDA-MB-231 (mean $\pm \mathrm{SE} ; \mathrm{n}=4-7 ;{ }^{*} \mathrm{p}<0.05 ;{ }^{* *} \mathrm{p}<0.01$ ). Data are presented as relative gene expression with respect to the housekeeping gene ACTB.

MCF7
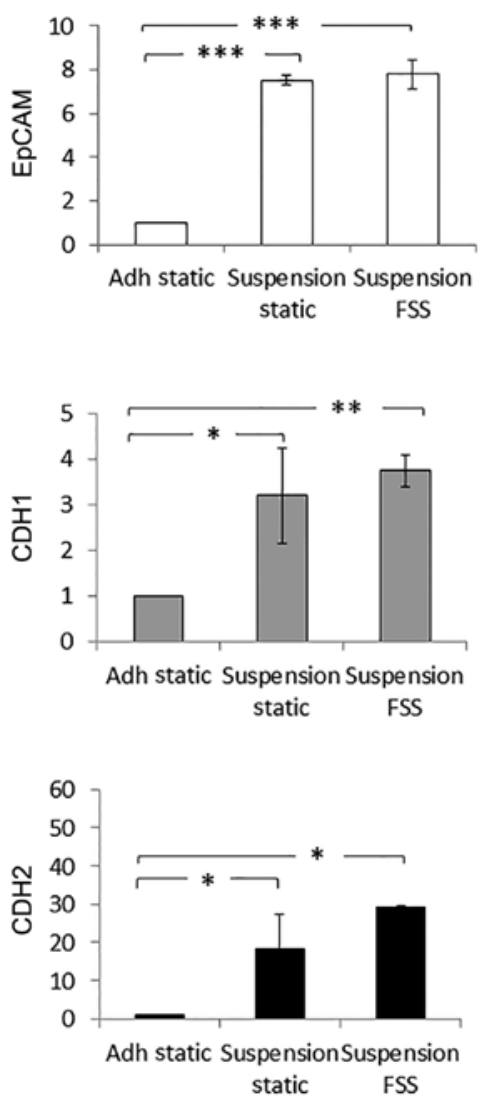

MDA-MB-231
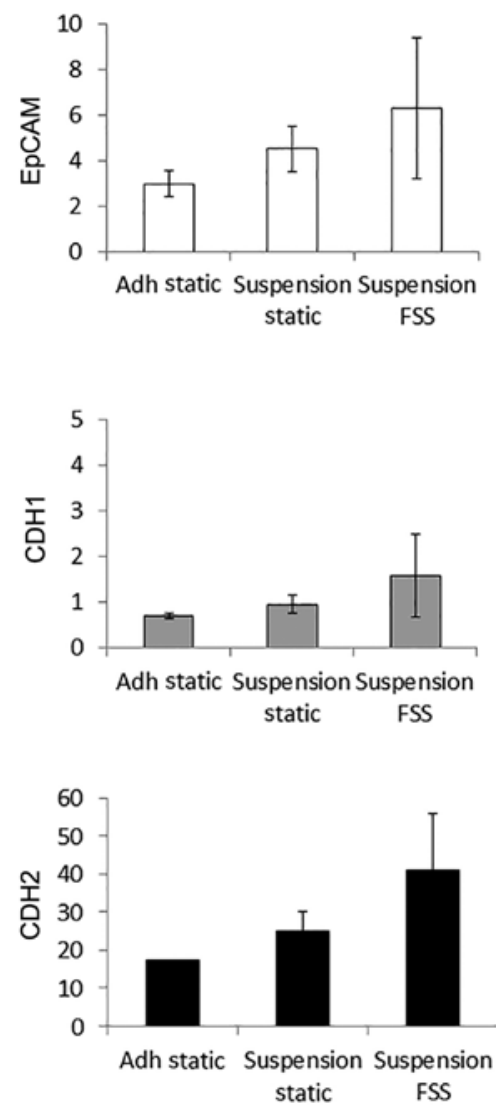

Figure 3. MCF7 cell line is more susceptible than MDA-MB-231 to changes in culture conditions. Quantitative RT-PCR was performed on MCF7 and MDAMB-231 cell lines grown in adherent and suspension culture conditions with and without fluid shear stress (FSS). EpCAM, CDH1, and CDH2 gene expression was significantly upregulated in MCF7 suspension culture (mean $\pm \mathrm{SD} ; \mathrm{n}=3 ;{ }^{*} \mathrm{p}<0.05 ;{ }^{* * *} \mathrm{p}<0.01 ;{ }^{* * * *} \mathrm{p}<0.001$ ). Data are presented as relative gene expression with respect to the housekeeping gene ACTB. 
A

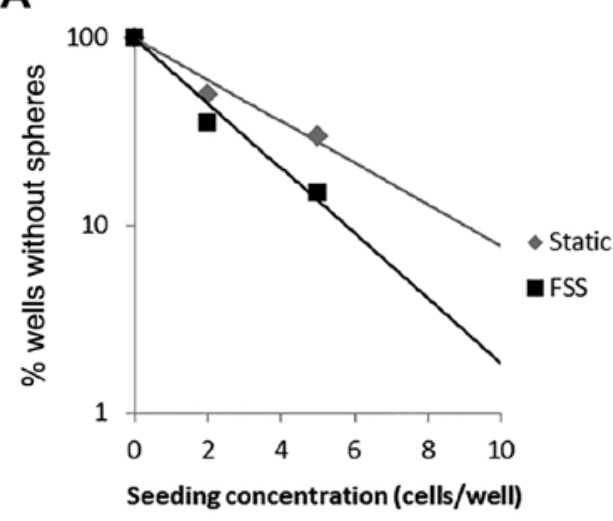

B

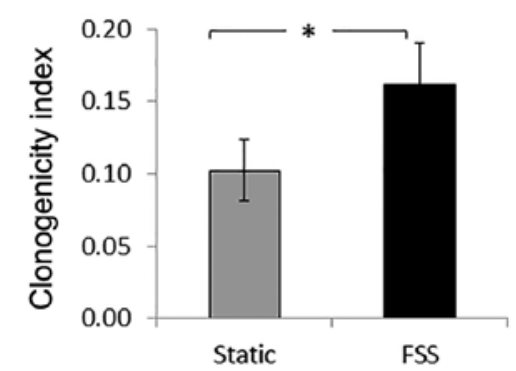

MDA-MB-231
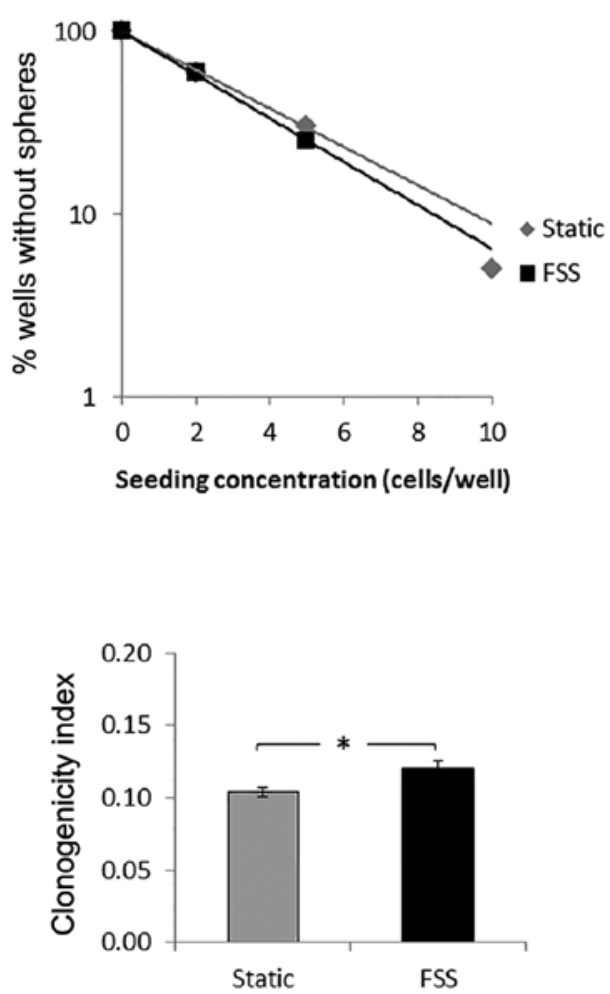

Figure 4. MCF7 is more clonogenic than MDA-MB-231 cell line. Limiting dilution assays (LDAs) were used to test clonogenicity of MCF7 and MDA-MB-231 suspension static and suspension fluid shear stress (FSS) cultures. (A) Linear regression of MCF7 and MDA-MB-231 showed less number of cells without spheres for FSS cultures. Steeper slope indicates higher clonogenicity. (B) Clonogenicity index showed a larger increase in clonogenicity for suspension FSS MCF7 than suspension FSS MDA-MB-231 cell line (Clonogenicity index: slope \pm SE of linear regression of LDAs; ${ }^{*}$ p<0.05).

both cells, but there was a higher increase of clonogenicity with MCF7 cells at 1.6-fold (vs. 1.2 for MDA-MB-231). Changes in stem cell marker expression upon FSS were further explored using the stemness markers NANOG and OCT4. A higher stemness expression was observed in MCF7 suspension culture than in adherent cultured cells (Fig. 5A and B). There was a further increase in the stemness marker expression upon FSS at both protein and transcript levels, corroborating the data seen with the LDAs. No increase of the stemness marker expression was seen in the MDA-MB-231 western blot data, though there was a significant increase observed for the OCT4 transcript (Fig. 5B). However, the fold change for OCT4 was much greater in MCF7 than in MDA-MB-231.

As a more specific measure of breast CSC expression, surface protein expression of the CD24 and CD44 markers was tested with MCF7 and MDA-MB-231 in adherent static, suspension static, and suspension FSS conditions. MCF7 CD $44^{+} / \mathrm{CD} 24^{-}$expression increased significantly from the adherent culture to suspension static culture with approximately a $25 \%$ increase in expression (from $7 \%$ expression to $31 \%$ expression, respectively) (Fig. 5C). This was expected as serum-free culture is known to concentrate CSCs $(15,24)$. CD $44^{+} / \mathrm{CD} 24^{-}$expression also increased in MCF7 suspension static to suspension FSS conditions from 31 to $46 \%$, respectively. This showed that MCF7 cells can be induced to a higher CSC population by changes in culture conditions from adherent to suspension and under FSS conditions. The MDA-MB-231 cell line showed no changes in CD44 $/$ CD24- expression and remained consistently high at over $80 \%$ expression in all three samples.

Since EpCAM is a clinically used surface protein marker for CTCs, EpCAM expression was tested using flow cytometry. As seen with qRT-PCR, MCF7 had a higher overall EpCAM expression than MDA-MB-231 (Fig. 5D). There was an increase in EpCAM for MCF7 suspension FSS cells from the static conditions. However, only $7 \%$ of the MDA-MB-231 expressed EpCAM in the adherent static conditions. This dropped to 1.57 and $1.13 \%$ in the suspension static and suspension FSS cells, respectively.

\section{Discussion}

The changes in cancer cell characteristics are important in understanding the metastatic process. However, cancer metastasis is difficult to model in experimental research. Most research relies on animal models to study tumor formation and distant colonization $(25,26)$. These studies provide an ultimate outcome when metastasis occurs, but provide little insight on the processes. Although this study did not focus on the direct mechanism of FSS on metastatic potential, there have been previous studies of the effects of FSS in the biological pathway Tie $2(9,27,28)$. One study focused on the Tie 2 pathway where FSS as high as 20 dyne $/ \mathrm{cm}^{2}$ was found to activate Tie2 and PI3K/Akt phosphorylation in endothelial cells (28). Furthermore, PI3K/Akt activation has been seen in ovarian carcinoma under low FSS condition (9) and 
A

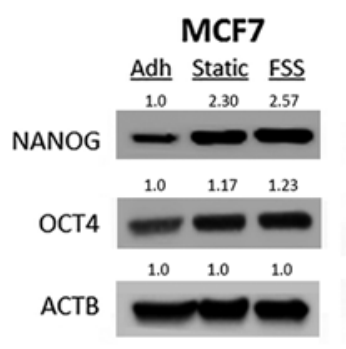

B
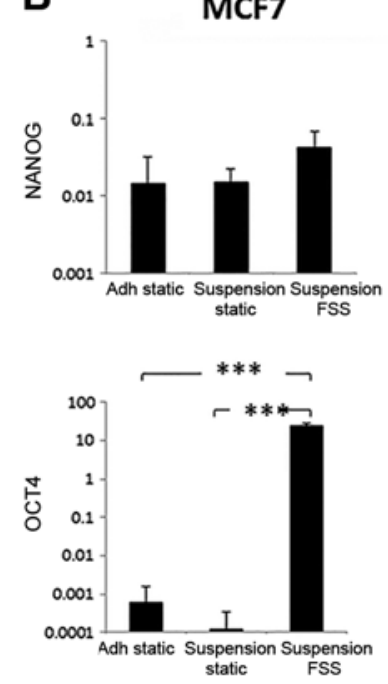

MDA-MB-231

$\underline{\text { Adh }} \underline{\text { Static } \underline{\text { FSS }}}$

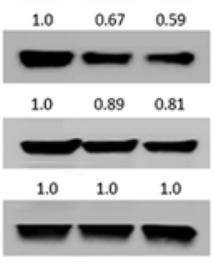

MDA-MB-231
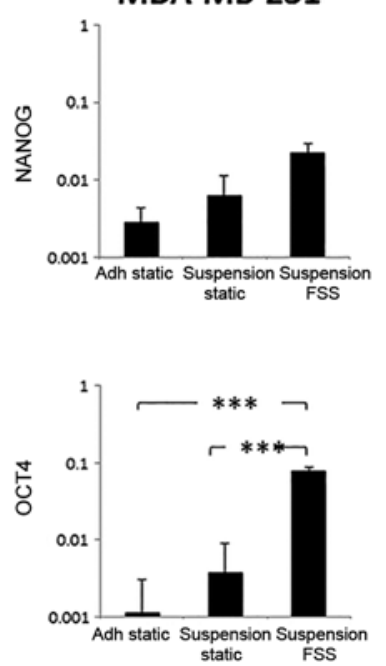

C

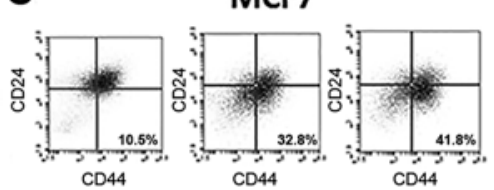

MCF7

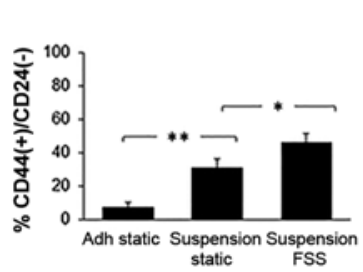

D $\quad$ MCF7

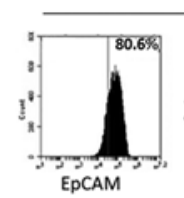

MCF7
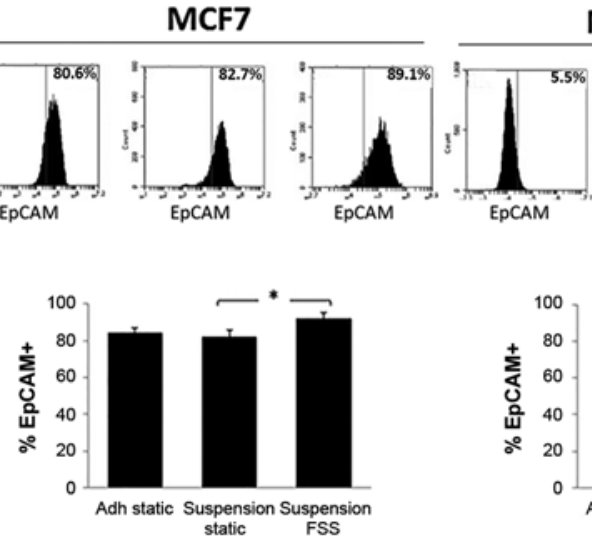

MDA-MB-231

MDA-MB-231
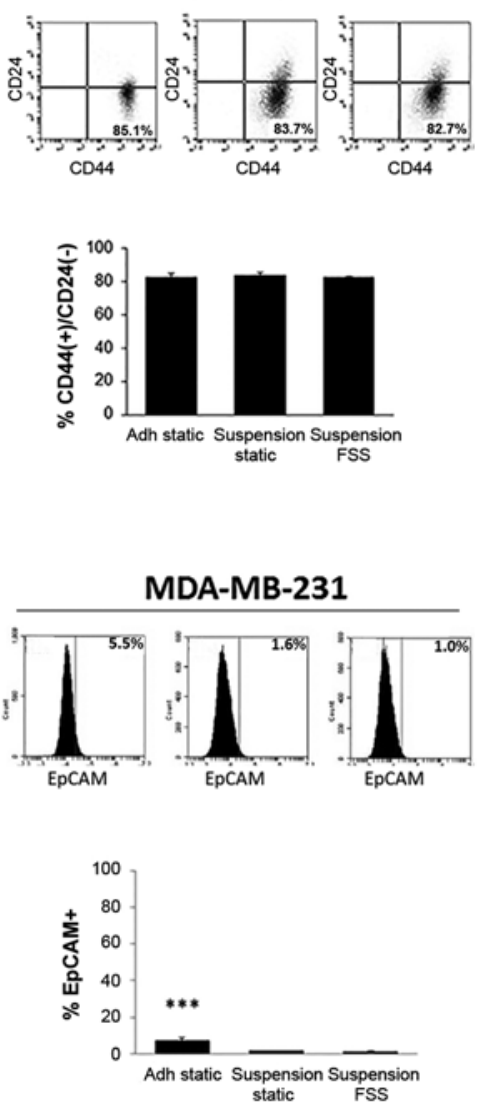

Figure 5. MCF7 cell line is more susceptible than MDA-MB-231 to CSC expression changes. Western blotting and qRT-PCR was used to analyze the NANOG and OCT4 stemness marker expression in adherent and suspension cultured conditions with and without fluid shear stress (FSS) (A and B). Flow cytometry was used to analyze $\mathrm{CD} 44^{+} / \mathrm{CD} 24^{-} \mathrm{CSC}$-like population of cells and $\mathrm{EpCAM}^{+}$population of cells in adherent and suspension culture conditions with and without FSS (C and D). (A) Western blots of MCF7 showed an increase in stemness in suspension culture from adherent culture. MCF7 also showed an increase in suspension FSS culture from suspension static culture. MDA-MB-231 showed no increase in stemness. (B) qRT-PCR showed a significant increase in suspension FSS OCT4 expression for both cell lines (mean $\pm \mathrm{SD} ; \mathrm{n}=3 ;^{* * * *} \mathrm{p}<0.001$ ). (C) MCF7 had a significant increase in the percentage of $\mathrm{CD}^{\circ} 4^{+} / \mathrm{CD}^{-} 4^{-}$expression in suspension culture than in adherent culture. MCF7 also had a significant increase in the percentage of CD44 $/$ CD24- expression under FSS. There was no significant change in CD44 /CD24- expression for MDA-MB-231. (D) MCF7 had a high level of EpCAM in adherent, suspension static, and suspension FSS cultures. MDA-MB-231 had a low level of EpCAM expression where the adherent culture cells had a significantly higher expression than the suspension culture $\left(\right.$ mean $\left.\pm \mathrm{SD} ; \mathrm{n}=3 ;{ }^{*} \mathrm{p}<0.05 ;{ }^{* *} \mathrm{p}<0.01 ;{ }^{* * *} \mathrm{p}<0.001\right)$.

most recently has been reported that Tie2 regulates stemness and metastatic properties in prostate cancer (27). However, metastasizing cancer cell properties have not been thoroughly studied under the effects of fluid shear stress (FSS). Blood flow and its components can have both a negative and positive effect on circulating tumor cells (CTCs). Studies have shown CTC-platelet adhesion aids in CTC survival by protecting them from FSS $(29,30)$. CTCs have also been seen to have higher viability under FSS than their non-cancerous counterparts (Fig. 1) (7,31). Therefore, these previous reports and our data motivated us to examine EMT, CTC, and CSC marker expression of cells under FSS as a model for metastasis.

Since EMT is an important first step of metastasis, it was hypothesized that FSS can induce EMT expression in breast cancer cell lines. An increase in mesenchymal gene expression and a decrease in epithelial gene expression were originally expected in MCF7 and MDA-MB-231. In this study, however, we did not observe a decrease in epithelial gene expression. Nevertheless, EMT gene expression (i.e. mesenchymal) was high in non-adherent cells. Other models have shown that cells do not need to undergo complete EMT in order to metastasize to secondary tumors $(32,33)$. Previous studies have shown that $\mathrm{CDH} 2$ can promote motility in certain breast cell lines regardless of high $\mathrm{CDH} 1$ expression (34). It is possible that only a partial EMT is occurring where cells maintain their epithelial characteristics while still undergoing upregulation of mesenchymal markers.

Based on the above results, we proposed an in vitro model of metastasis that incorporated different culture conditions and FSS (Fig. 6). Adherent static cultured cells can be thought to represent cancer cells in the solid primary tumor due to the solid tumor stiff epithelial morphology. Suspension cultured cells are likely models of cells detaching from the primary tumor. CTCs were then modeled as suspension cultured cells under FSS. Testing the EpCAM, CDH1, and CDH2 markers showed that our 'detached' cells were the most prone to EMT expression and that FSS did not attenuate this response.

Cancer stem cells (CSCs) have been suggested as an important factor contributing to metastasis. As a test of stemness and proliferation, clonogenicity via limiting dilution assay was used on suspension static and suspension FSS cells. A significant increase in clonogenicity was seen in 


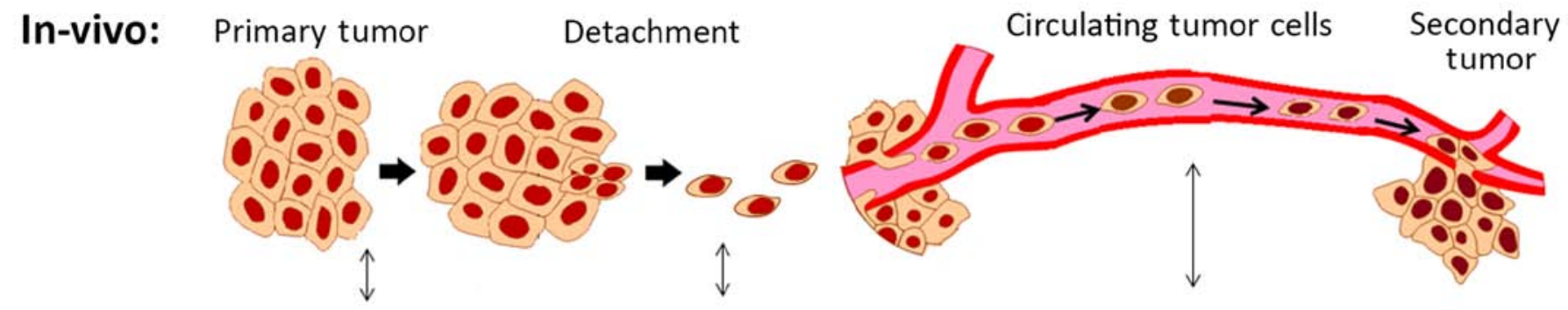

In-vitro model: Adherent cells

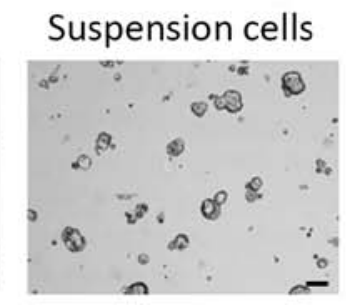

Suspension cells in FSS

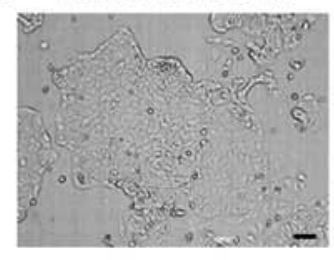

Figure 6. Model of metastasis. Adherently cultured cells are thought as models of primary tumors, whereas suspension cultured cells are thought to be models of detaching tumor cells undergoing EMT. These detached suspension cells then undergo FSS in the blood vessels as CTCs. Results from our study with MCF7, shown as micrographs of in vitro model, suggest that these detaching primary tumor CTCs acquire CSC-like characteristics under FSS.

both MCF7 and MDA-MB-231 suspension FSS compared to suspension static culture (Fig. 4). MCF7 had a higher increase in clonogenicity of suspension FSS from static conditions in comparison to MDA-MB-231. This was further corroborated with NANOG and OCT4 stemness marker expression where MCF7 increased in suspension FSS compared to suspension static culture (Fig. 5A). Similar to the LDAs, OCT4 significantly increased in suspension FSS culture for both cell lines but, a larger fold increase was seen in MCF7 than MDA-MB-231 (Fig. 5B).

Breast CSC markers were tested under FSS. The individual marker expression for CD24 and CD44 was initially tested from cells under FSS. As expected and seen in other reports (35), the less invasive MCF7 had an overall lower $\mathrm{CD} 44^{+}$population and higher $\mathrm{CD} 24^{+}$. In contrast, the highly invasive MDA-MB-231 cell line had over $90 \%$ CD44+ expression (data not shown). Furthermore, the effect of FSS on CSC-like CD $44^{+} / \mathrm{CD} 24^{-}$population was tested on adherent and suspension cultured cells. Overall, MDA-MB-231 had a consistently high CSC signature that did not change with FSS or culture conditions. However, the CSC signature increased in the suspension culture and in FSS conditions for MCF7 (Fig. 5D), which corroborates the correlation between CTCs and CSCs previously seen in cells undergoing EMT $(36,37)$. Furthermore, our data also showed that FSS induced CSC-like expression in the MCF7 cell line.

Our data demonstrated that the MCF7 cell line was overall more susceptible to changes in EMT and CSC expression. Although FSS induced an increase in CSC expression, the culture conditions of the cell line had a larger impact on expression changes. However, the MDA-MB-231 cell line was more resistant to changes in both gene and protein expression. This is most likely due to differences in the cell line characteristics. Breast cancer can be divided into the following four subtypes in order of increasing invasiveness and severity: luminal A, luminal B, human epidermal growth factor receptor 2 (HER2)positive and triple-negative (TNBC) $(38,39)$. Dividing breast cancer into these subtypes has allowed for better characterization according to their genetic makeup. These subtypes can also be characterized by the expression status of three key cell surface hormone receptor proteins: estrogen receptor (ER), progesterone receptor (PR), and HER2. Luminal A breast cancers are $\mathrm{ER}^{+} /$ $\mathrm{PR}^{+} / \mathrm{HER} 2^{-}$, and triple-negative are ER/PR/HER2-. MCF7, a non-invasive breast cancer, belongs to the luminal A subtype, while MDA-MB-231, an invasive breast cancer, belongs to the TNBC subtype (40). MDA-MB-231, which is already highly invasive and more aggressive as seen in both EMT and CSC gene expression, is therefore more resistant to changes in culture conditions and FSS.

In conclusion, the importance of cancer metastasis has resulted in a variety of new detection devices and treatment options; however, the effects of FSS on cancer cells have not been exclusively studied. Our study addresses these issues by using an in vitro model of FSS on breast cancer cells. Herein, we found that the effect of FSS is dependent on breast cancer subtype. Culture conditions also had a large effect on EMT/CTC and CSC expression which was used as a model for metastasis (Fig. 6).

\section{Acknowledgements}

We are grateful to Dr John W. Van Zee and Dr Matthew Jenny for providing equipment support. We are also grateful to Ria Corder and Brittney Sunday for providing technical assistance, and to Dr Dong Woon Kim for his critical reading and input to our manuscript. This material is based upon work supported by the National Science Foundation (NSF) under grant no. 1342388 and 1604677 . N.L.K. was a participant of the Research Experience for Undergraduates (REU) at the University of Alabama, supported through the NSF REU Site grant no. 1358750 .

\section{References}

1. Chaffer CL and Weinberg RA: A perspective on cancer cell metastasis. Science 331: 1559-1564, 2011. 
2. Dong Y, Skelley AM, Merdek KD, Sprott KM, Jiang C, Pierceall WE, Lin J, Stocum M, Carney WP and Smirnov DA: Microfluidics and circulating tumor cells. J Mol Diagn 15: 149-157, 2013

3. Li P, Stratton ZS, Dao M, Ritz J and Huang TJ: Probing circulating tumor cells in microfluidics. Lab Chip 13: 602-609, 2013.

4. Friedlander TW, Premasekharan G and Paris PL: Looking back, to the future of circulating tumor cells. Pharmacol Ther 142: 271-280, 2014

5. Broersen LH, van Pelt GW, Tollenaar RA and Mesker WE: Clinical application of circulating tumor cells in breast cancer. Cell Oncol (Dordr) 37: 9-15, 2014.

6. Mitchell MJ and King MR: Computational and experimental models of cancer cell response to fluid shear stress. Front Oncol 3: 44, 2013

7. Barnes JM, Nauseef JT and Henry MD: Resistance to fluid shear stress is a conserved biophysical property of malignant cells PLoS One 7: e50973, 2012.

8. Aktas B, Tewes M, Fehm T, Hauch S, Kimmig R and Kasimir-Bauer S: Stem cell and epithelial-mesenchymal transition markers are frequently overexpressed in circulating tumor cells of metastatic breast cancer patients. Breast Cancer Res 11: R46, 2009.

9. Ip CK, Li SS, Tang MY, Sy SK, Ren Y, Shum HC and Wong AS Stemness and chemoresistance in epithelial ovarian carcinoma cells under shear stress. Sci Rep 6: 26788, 2016.

10. Nguyen LV, Vanner R, Dirks P and Eaves CJ: Cancer stem cells An evolving concept. Nat Rev Cancer 12: 133-143, 2012.

11. Reya T, Morrison SJ, Clarke MF and Weissman IL: Stem cells, cancer, and cancer stem cells. Nature 414: 105-111, 2001.

12. Al-Hajj M, Wicha MS, Benito-Hernandez A, Morrison SJ and Clarke MF: Prospective identification of tumorigenic breast cancer cells. Proc Natl Acad Sci USA 100: 3983-3988, 2003.

13. Ginestier C, Hur MH, Charafe-Jauffret E, Monville F, Dutcher J, Brown M, Jacquemier J, Viens P, Kleer CG, Liu S, et al: ALDH1 is a marker of normal and malignant human mammary stem cells and a predictor of poor clinical outcome. Cell Stem Cell 1: 555-567, 2007.

14. Visvader JE and Lindeman GJ: Cancer stem cells in solid tumours: Accumulating evidence and unresolved questions. Nat Rev Cancer 8: 755-768, 2008.

15. Ponti D, Costa A, Zaffaroni N, Pratesi G, Petrangolini G, Coradini D, Pilotti S, Pierotti MA and Daidone MG: Isolation and in vitro propagation of tumorigenic breast cancer cells with stem/progenitor cell properties. Cancer Res 65: 5506-5511, 2005.

16. Liao M-J, Zhang CC, Zhou B, Zimonjic DB, Mani SA, Kaba M, Gifford A, Reinhardt F, Popescu NC, Guo W, et al: Enrichment of a population of mammary gland cells that form mammospheres and have in vivo repopulating activity. Cancer Res 67: 8131-8138, 2007.

17. Livak KJ and Schmittgen TD: Analysis of relative gene expression data using real-time quantitative PCR and the 2(- $\Delta \Delta \mathrm{C}(\mathrm{T}))$ method. Methods 25: 402-408, 2001

18. Haley EM, Tilson SG, Triantafillu UL, Magrath JW and Kim Y: Acidic $\mathrm{pH}$ with coordinated reduction of basic fibroblast growth factor maintains the glioblastoma stem cell-like phenotype in vitro. J Biosci Bioeng: Jan 4, 2017 (Epub ahead of print).

19. Mallini P, Lennard T, Kirby J and Meeson A: Epithelial-tomesenchymal transition: What is the impact on breast cancer stem cells and drug resistance. Cancer Treat Rev 40: 341-348, 2014.

20. Lamouille S, Xu J and Derynck R: Molecular mechanisms of epithelial-mesenchymal transition. Nat Rev Mol Cell Biol 15: 178-196, 2014

21. Onder TT, Gupta PB, Mani SA, Yang J, Lander ES and Weinberg RA: Loss of E-cadherin promotes metastasis via multiple downstream transcriptional pathways. Cancer Res 68 : 3645-3654, 2008.
22. van Roy F: Beyond E-cadherin: Roles of other cadherin superfamily members in cancer. Nat Rev Cancer 14: 121-134, 2014.

23. Schnell U, Cirulli V and Giepmans BN: EpCAM: Structure and function in health and disease. Biochim Biophys Acta 1828: 1989-2001, 2013.

24. Yuan X, Curtin J, Xiong Y, Liu G, Waschsmann-Hogiu S, Farkas DL, Black KL and Yu JS: Isolation of cancer stem cells from adult glioblastoma multiforme. Oncogene 23: 9392-9400, 2004.

25. Bos PD, Nguyen DX and Massagué J: Modeling metastasis in the mouse. Curr Opin Pharmacol 10: 571-577, 2010.

26. Saxena $M$ and Christofori G: Rebuilding cancer metastasis in the mouse. Mol Oncol 7: 283-296, 2013.

27. Tang KD, Holzapfel BM, Liu J, Lee TK, Ma S, Jovanovic L, An J, Russell PJ, Clements JA, Hutmacher DW, et al: Tie-2 regulates the stemness and metastatic properties of prostate cancer cells. Oncotarget 7: 2572-2584, 2016

28. Lee HJ and Koh GY: Shear stress activates Tie2 receptor tyrosine kinase in human endothelial cells. Biochem Biophys Res Commun 304: 399-404, 2003.

29. Green DL and Karpatkin S: Effect of cancer on platelets. In: Coagulation in Cancer. Kwaan $\mathrm{CH}$ and Green D (eds). Springer, Boston, MA, pp17-30, 2009.

30. Gay LJ and Felding-Habermann B: Contribution of platelets to tumour metastasis. Nat Rev Cancer 11: 123-134, 2011.

31. Mitchell MJ, Denais C, Chan MF, Wang Z, Lammerding J and King MR: Lamin A/C deficiency reduces circulating tumor cell resistance to fluid shear stress. Am J Physiol Cell Physiol 309: C736-C746, 2015.

32. Christiansen JJ and Rajasekaran AK: Reassessing epithelial to mesenchymal transition as a prerequisite for carcinoma invasion and metastasis. Cancer Res 66: 8319-8326, 2006.

33. Reymond N, d'Água BB and Ridley AJ: Crossing the endothelial barrier during metastasis. Nat Rev Cancer 13: 858-870, 2013.

34. Nieman MT, Prudoff RS, Johnson KR and Wheelock MJ: $\mathrm{N}$-cadherin promotes motility in human breast cancer cells regardless of their E-cadherin expression. J Cell Biol 147: 631-644, 1999.

35. Ricardo S, Vieira AF, Gerhard R, Leitão D, Pinto R, CameselleTeijeiro JF, Milanezi F, Schmitt F and Paredes J: Breast cancer stem cell markers CD44, CD24 and ALDH1: Expression distribution within intrinsic molecular subtype. J Clin Pathol 64: 937-946, 2011.

36. Mani SA, Guo W, Liao M-J, Eaton EN, Ayyanan A, Zhou AY, Brooks M, Reinhard F, Zhang CC, Shipitsin M, et al: The epithelial-mesenchymal transition generates cells with properties of stem cells. Cell 133: 704-715, 2008.

37. Liu S, Cong Y, Wang D, Sun Y, Deng L, Liu Y, Martin-Trevino R, Shang L, McDermott SP, Landis MD, et al: Breast cancer stem cells transition between epithelial and mesenchymal states reflective of their normal counterparts. Stem Cell Rep 2: 78-91, 2013.

38. Sanpaolo P, Barbieri V and Genovesi D: Prognostic value of breast cancer subtypes on breast cancer specific survival, distant metastases and local relapse rates in conservatively managed early stage breast cancer: A retrospective clinical study. Eur J Surg Oncol 37: 876-882, 2011.

39. Paquet ER and Hallett MT: Absolute assignment of breast cancer intrinsic molecular subtype. J Natl Cancer Inst 107: dju357, 2015

40. Holliday DL and Speirs V: Choosing the right cell line for breast cancer research. Breast Cancer Res 13: 215, 2011. 